\title{
RENDIMIENTO ACADÉMICO Y ESPECIALIDAD PREVIA DE LOS ESTUDIANTES DE PSICOPEDAGOGÍA DEL C.E.S. DON BOSCO DE MADRID
}

\author{
EDUCATIONAL ACHIEVEMENT AND PREVIOUS ACADEMIC \\ BACKGROUND OF STUDENTS OF PSYCHO-PEDAGOGY AT \\ «DON BOSCO» COLLEGE IN MADRID
}

\author{
J. Quintanal, B. García, J. C. Sánchez y L. Pérez \\ Universidad Complutense de Madrid
}

\begin{abstract}
RESUMEN
En la actualidad, la licenciatura en Psicopedagogía está concebida como una carrera de segundo ciclo que viene a completar una titulación de grado medio en alguna especialidad previa relacionada con la educación. El hecho de que ésta última pudiera ser tan diversa nos hizo pensar en la posibilidad de que pudiera existir alguna especialidad de la que procedieran los alumnos con mejores resultados y, de ser así, si esto sugeriría la necesidad de efectuar algún tipo de reajuste en los programas y planes de estudio. Para responder a estas cuestiones, analizamos las calificaciones obtenidas por el grupo de alumnos de primer curso de Psicopedagogía matriculados durante el curso escolar 1999/2000 en el C.E.S. Don Bosco de Madrid, poniéndolas en relación con su formación de origen. Los resultados mostraron que no existen diferencias de consideración entre los distintos subgrupos comparados y, por tanto, no parece que pueda concluirse que exista relación entre el resultado académico obtenido en las asignaturas del plan de estudios de la licenciatura y la formación precedente por la que se accede a ellas, por lo que no hay datos que apoyen la idea de que ésta pueda utilizarse para predecir el futuro éxito o fracaso de nuestros estudiantes.
\end{abstract}

Palabras clave: Rendimiento académico, Psicopedagogía.

\begin{abstract}
The degree in Education and Psychology conforms a complex corpus coming from basics of the First Cycle (in an Educational field), and a Second Cycle formed by a program guided to the specific professional development in this context of behaviour. The fact that the previous curricular specialization of these students could be so diverse has encouraged us to wonder for the predictable value that this could have on the academic results in the degree. From what field do the students come with better results?. Would it be possible some type of readjustment in programs and study plans which would allow us to optimize the effect of the formation on the specific matters of these studies? To reply to
\end{abstract}


these questions, we analyze the qualifications obtained by the group of students of the First Course on Education and Psychology registered during the 1999/2000 academic course in the C.E.S. «Don Bosco», Madrid, with the objective of establishing possible problems between them and the formation of the First Cycle of the mentioned group. The results showed that no considerable differences exist among the different subgroups already compared. So, it cannot be concluded that there is a relationship between the academic result obtained in the subjects of the degree and the previous formation. Neither that this can be used, beforehand, to predict the success or failure in the future of our students.

Key words: Educational achievement, Psycho-pedagogy.

\section{Introducción}

Los estudios de Psicopedagogía, de reciente implantación en nuestro sistema educativo, conforman un corpus sistémico complejo resultado de la formación básica en el primer ciclo universitario de alguna especialidad relacionada con la Educación y una fase posterior de segundo ciclo en la que se completa esta formación con un programa específicamente orientado al desarrollo profesional del orientador. De este modo, el hecho de que el alumnado que cursa esta licenciatura pudiera tener diversa formación académica nos hizo preguntarnos si existía alguna especialidad en particular de la que procedieran los alumnos con mejores resultados en segundo ciclo y, de ser así, si esto podría sugerir la necesidad de plantear algún tipo de reajuste en los planes de estudio que permitiera optimizar el efecto de los llamados «complementos de formación» que dan paso a la obtención del título de Licenciado.

Para dar respuesta a ambas cuestiones, desarrollamos el estudio que aquí presentamos apoyándonos en los resultados académicos obtenidos durante el curso escolar 1999/2000 por un grupo de alumnos de primer curso de Psicopedagogía del C.E.S. Don Bosco de Madrid (adscrito a la Universidad Complutense).

\section{Justificación}

Como decimos, los estudios de Psicopedagogía tienen una vida aún efímera en el marco de nuestro Sistema Educativo (Legislación, 1992). Por lo que supone en términos de diseño e implantación puede decirse que es una realidad muy reciente, fruto de la evolución natural que ha seguido la educación a lo largo del siglo XX (Monereo y Solé, 1999), que pretende afianzarse en el panorama formativo actual con una identidad propia con la que contribuir al desarrollo de la acción educativa.

En los términos evolucionistas a los que nos hemos referido, Coll (1999, 34-41) plantea con detalle cómo esta disciplina ha ido integrándose en la Universidad como respuesta a esa necesidad que aparece con la nueva concepción que se tiene de la educación: integradora y diversificadora a la vez, generadora de expectativas y potenciadora de relaciones interpersonales, dirigida al sujeto en particular pero velando por su adecuada socialización...

De este modo, y con el objeto de adaptarse a esta nueva realidad, la Psicopedagogía aparece vinculada a todos los cambios que presenta el sistema educativo, lo que hace que el profesional dedicado a ella se interese y preocupe de manera especial por la nueva estructura y organización que éste presente. Así, su diseño curricular se nos muestra como respuesta a un marco concreto (el de esas necesidades que surgen con el nuevo planteamiento de la 
educación), que intenta ofrecer soluciones adecuadas a la necesidad de orientación que el sujeto de la nueva sociedad requiere. Eso sí, respetando siempre el estilo de actuación que impone la nueva relación educativa en la que, como destacan Repetto, Ballesteros y Málik (2000), se confiere mayor importancia «al aspecto práctico de la formación», lo que nos obliga, como formadores de esos futuros orientadores-psicopedagogos, a plantear un sistema de formación profesionalizado, coherente y debidamente estructurado.

Analizarlo no es objeto específico de nuestro estudio, aunque reconocemos la necesidad que actualmente puede plantear la revisión de los planes formativos que al respecto están vigentes. Más bien, y teniendo presente que el tema de la calidad es una de las cuestiones que con mayor intensidad se le está exigiendo a la Didáctica y a la Organización Escolar (preocupación que, como señala la OCDE (1991), citado por Gento $(2000,481)$, ha de producirse fundamentalmente en los centros e instituciones educativas), queremos trasladar esta reflexión al ámbito universitario, en concreto, a nuestro Centro de Enseñanza Superior «Don Bosco» de Madrid (Universidad Complutense) que oferta, entre sus titulaciones, la formación en Psicopedagogía desde el curso 1999/2000, según el plan de estudios de 1995 aprobado por esta Universidad (Legislación 1994, revisado en 1998).

Entre los predictores que según Gento $(2000,510)$ pueden afectar a dicha calidad educativa, se incluye el perfil formativo que se adquiere antes de cursar cualquier licenciatura. Dado que el propio Ministerio de Educación (MEC, 1994) insiste en este hecho, nos parece oportuno analizar en qué medida la formación inicial (primer ciclo) de los futuros psicopedagogos puede afectar a la calidad de su labor profesional. En otras palabras, y a tenor de las distintas opciones que la legislación oficial (1992) establece para integrarse en los estudios de Psicopedagogía (haber completado estudios de primer ciclo en cualquier de las especialidades de Magisterio, de Educación Social, Pedagogía o Psicología), nos preguntamos si existe algún tipo de correlación entre la procedencia formativa de nuestros alumnos y los resultados académicos que obtengan.

Con este objetivo inicial, pretendemos ahondar en lo que propone Municio $(2000,477)$ cuando señala que «el planteamiento de la calidad no es más que realizar un cuidadoso estudio previo para ajustar el proceso (como conjunto) y establecer cuáles son las respuestas probables de las personas»; o, como bien indica el profesor Pérez Juste $(2000,565)$, «los programas educativos son realidades complejas; su correcta formulación, su adecuación a los destinatarios, el cumplimiento de las exigencias técnicas... son aspectos sobre los que se puede aprender, como consecuencia de la acumulación de datos y conclusiones a partir de evaluaciones de programas». En definitiva, en este trabajo nos planteamos el doble objetivo de reflexionar sobre los fundamentos que puedan sustentar la esperanza de calidad con que nos enfrentemos a nuestro alumnado y de contribuir a predecir sus necesidades formativas optimizando los programas de estudio en virtud de las mismas.

\section{Objetivos}

Concretamente, y a tenor de lo desarrollado en los apartados anteriores, nos planteamos como objetivo general analizar los resultados obtenidos por nuestros alumnos de Psicopedagogía, en virtud del origen académico de sus estudios de primer ciclo, con el ánimo de sugerir mejoras relativas a los complementos de formación más adecuados a cada una de las rutas de acceso previstas. Más específicamente, pretendemos: 
- Describir los resultados obtenidos por nuestros alumnos en virtud de su ruta formativa precedente y determinar la existencia de diferencias significativas entre ellos atendiendo a su especialización previa.

- En caso de encontrar diferencias, calcular el valor de dicha especialidad como factor predictor del éxito académico de los estudiantes de Psicopedagogía o, en su defecto, el grado de contingencia existente entre ambas variables.

- Y, por último, determinar en qué asignaturas concretas pueden presentan carencias aquellos alumnos procedentes de las especialidades con resultados inferiores, ofreciéndoles complementos de formación específicos en futuros planes de estudio.

\section{Hipótesis de trabajo}

En relación con los objetivos que acabamos de señalar, esperamos encontrar que:

- Los estudiantes de Psicopedagogía obtienen resultados académicos diferentes en virtud de la especialidad cursada en la ruta previa de acceso a los mismos.

- La especialización previa de los estudiantes de Psicopedagogía puede utilizarse como predictor de su éxito académico final.

- El análisis de resultados académicos de los alumnos de Psicopedagogía permite determinar las necesidades específicas que presentan los estudios de formación que dan acceso a la enseñanza de la especialidad en segundo ciclo.

\section{Metodología}

Para dar adecuada respuesta a los interrogantes reseñados, hemos llevado a cabo una investigación de carácter eminentemente básico que nos permita profundizar en el conocimiento de los estudios de Psicopedagogía. Para aproximarnos a esta realidad, planteamos desarrollar un estudio descriptivo perfectamente identificable dentro de la filosofía propia de la metodología correlacional.

\section{Muestra de estudio}

El presente trabajo se llevó a cabo a partir de los datos recogidos en una muestra de 123 alumnos de primer curso de Psicopedagogía del C.E.S. Don Bosco de Madrid. Dicha muestra se encontraba distribuida en dos turnos diferentes: 61 alumnos en el grupo de tarde $(49,6 \%)$ y 62 en el grupo de mañana $(50,4 \%)$. Atendiendo a su procedencia académica, la muestra quedó distribuida según se recoge en la figura 1 (ver Anexo).

Resulta conveniente señalar que, de las 21 opciones diferentes (materias) que conforman el Plan de Estudios establecido por la Universidad Complutense de Madrid en el año 95, tres de ellas determinan la especialidad formativa de cada alumno. Así, las asignaturas codificadas con los números 413, 414 y 415 en la tabla I establecen la especialidad de Orientación, mientras que la de Educación Especial viene dada por las materias cuyos códigos son el 410, 411 y 412. El resto de asignaturas, troncales u optativas, son comunes 
para ambas especialidades, si bien hemos de reseñar que los Complementos de formación que cursaron los alumnos de nuestra muestra de estudio fueron: para Educación Social las codificadas con los números 100, 101 y 102; para Magisterio (en cualquiera de sus especialidades) las identificadas bajo los códigos 100, 101, 102 y 103 y para Pedagogía las no 100,102 y 103.

\section{TABLA 1: Codificación numérica de las asignaturas de primer curso de Psicopedagogía del C.E.S. D. Bosco de Madrid (U.C.M.).}

\begin{tabular}{|l|l|}
\hline 100 - Psicometría & 408 - Estructura y Funcionamiento del Sistema Educativo \\
101 - Introducción a la Psicología & 409 - Aprendizaje Humano \\
102 - Psicología de la Personalidad & 410 - Historia de la Educación Especial \\
103 - Psicología Social & 411 - psicología de la Excepcionalidad \\
400 - Diagnóstico en Educación & 412 - Psicología clínica de la Educación \\
401 - Dificultades de Aprendizaje & 413 - Psicología Vocacional \\
402 - Modelos de Orientación & 414 - Psicología social de la Educación \\
403 - Métodos de Investigación & 415 - Orientación Personal \\
404 - Psicología de la Instrucción & 416 - Modelos de Comprensión Lectora \\
406 - Psicología de la Intervención & A01 - Etica de los Valores \\
407 - Psicopatología de la Infancia &
\end{tabular}

\section{Procedimiento}

Para realizar el estudio se procedió a la recogida de los siguientes datos de cada uno de los sujetos que componían la muestra:

- Nombre y apellidos

- DNI

- Turno (que como ya hemos indicado, puede ser de mañana o de tarde)

- Estudios de procedencia (en cuya reseña contemplamos la totalidad de especialidades de magisterio u otras especificadas en la figura 1 incluso con nomenclaturas no vigentes, pues algunos alumnos proceden de Planes antiguos).

- Calificación en las asignaturas cursadas en $1^{\circ}$ de Psicopedagogía (cuantificadas utilizando la nota final aparecida en las actas una vez finalizado el curso 1999/2000. A cada calificación le fue asignado el siguiente valor numérico: matrícula de honor (4), sobresaliente (3), notable (2), aprobado (1) y suspenso (0)).

\section{Análisis estadístico de los datos}

En un primer momento, los datos fueron sometidos a un tratamiento puramente descriptivo, para conocer las medias y desviaciones típicas de las calificaciones obtenidas por los 
alumnos de nuestra muestra en todas las asignaturas cursadas (tanto en términos globales como atendiendo a su especialidad de procedencia). Así mismo, obtuvimos las tablas y coeficientes de contingencia que relacionaban nuestra variable criterio (la especialidad de procedencia) con dichas calificaciones, proceso que nos permitió cuantificar el grado de asociación existente entre ellas.

Por último, y dado que nuestra variable criterio tenía más de dos niveles (diez, en concreto) y el número de alumnos asignado a cada uno de ellos era, en la mayoría de los casos, inferior a 30, utilizamos la prueba de contraste no paramétrica de Kruskal-Wallis, a un nivel de confianza del 95\%, para determinar la existencia de diferencias estadísticamente significativas tanto en las calificaciones globales como en las de cada asignatura en función de la especialización previa de los sujetos de la muestra.

\section{Resultados}

En términos generales, el estudio descriptivo de la calificación global media de nuestros alumnos, en virtud de su especialización previa, mostró unos rangos medios que oscilaron entre el 1,5 (entre aprobado y notable) alcanzado por el grupo de alumnos diplomados en Magisterio en su especialidad de Educación Física y el 2,11 (algo superior al notable) obtenido por el grupo de diplomados en la especialidad de Lengua Extranjera (ver figura 2 en el Anexo). Nótese que ha sido eliminada del análisis la especialidad de Pedagogía por falta de representatividad de la muestra, (tan sólo uno de nuestros alumnos estaba incluido en ella).

En cuanto a la dispersión de las puntuaciones conseguidas por los distintos grupos (medida a través de su desviación típica, s.d), se comprobó que la mayor variabilidad se dió entre los representantes de la especialidad de Educación Social $(\mathrm{s} . \mathrm{d}=1,27)$ y entre los que habían completado estudios en Planes Anteriores de Magisterio (s.d = 0,91), siendo los grupos más homogéneos el de Audición y Lenguaje y el de Educación Musical con desviaciones típicas de 0,38 y 0,27 , respectivamente. El resto de los grupos mostró un rango de dispersión medio de entre 0,5 y 0,75 .

Como podría desprenderse inicialmente de estos datos, las escasas diferencias observadas en estas calificaciones medias no ofrecieron significación estadística alguna entre ninguno de los grupos sometidos a comparación mediante la prueba de Kruskal-Wallis.

Sin embargo, el estudio individualizado de la relación de dependencia existente entre las distintas asignaturas cursadas por los sujetos de nuestra muestra y su especialidad académica de origen (mediante el cálculo de los respectivos coeficientes de contingencia), sí mostró cierto grado de asociación entre ellas, encontrándose que seis de las asignaturas estuvieron significativamente relacionadas con los estudios previos de los sujetos de nuestra muestra. Así, para las asignaturas Psicología de la Personalidad (coeficiente de contingencia significativo $=0,53$ ), Modelos de Orientación (coeficiente de contingencia significativo $=0,58$ ), Psicopatología de la Infancia (coeficiente de contingencia significativo =0,64), Estructura y Funcionamiento del Sistema Educativo (coeficiente de contingencia significativo = 0,63), Aprendizaje Humano (coeficiente de contingencia significativo =0,54) y Psicología Social de la Educación (coeficiente de contingencia significativo $=0,64$ ), sí podría pensarse que resultaron importantes los conocimientos adquiridos en sus estudios universitarios de primer grado. 
En este sentido, el análisis a posteriori de los datos medios incluidos en la tabla II, parece señalar que el hecho de haber cursado previamente la especialidad de Audición y Lenguaje facilita el éxito en Psicología de la Personalidad (media = 2,05), mientras que los alumnos procedentes de Educación Física encontrarán más complicaciones en ella (media $=1,33$ ). Por su parte, y en lo relativo a los Modelos de Orientación, los diplomados en Educación Física y Educación Social parecen no adaptarse bien a esta asignatura $($ media $=1,00)$, mientras que los procedentes de Educación Especial (media $=2,33)$, Lengua Extranjera (media $=2,29)$ y, sobre todo, los alumnos de Magisterio de planes anteriores (media $=2,67)$, obtienen óptimos resultados. En Psicopatología de la Infancia, de nuevo se repiten las diferencias entre los extremos representados por el grupo de Educación Social $($ media $=1,00)$ y el de los diplomados en planes anteriores de Magisterio (media $=2,67$ ). La Estructura y Funcionamiento del Sistema Educativo parece especialmente complicada para los especialistas en Educación Física (media $=0,60$ ), destacando muy por encima las calificaciones de los grupos de Educación Musical y Lengua Extranjera (media = 2,50). En la asignatura de Aprendizaje Humano vuelve a destacar el grupo de Educación Musical (media $=2,50)$, siendo en esta ocasión los diplomados en Educación Infantil los que presentaron peores resultados. Por último, las altas calificaciones obtenidas por todos los grupos en Psicología Social de la Educación, mostraron bastante divergencia entre los especialistas en Educación Física (media = 1,60) y los diplomados en planes anteriores de Magisterio.

Para finalizar con el análisis de los datos incluidos en esta tabla, tan sólo resaltar que, si nos fijamos en la primera de sus columnas en la que se incluye la media general obtenida en cada asignatura, podremos comprobar que aquellas que resultaron «más difíciles» para el total de la muestra fueron el Aprendizaje Humano (media = 1,23), seguido muy de cerca por las Dificultades de Aprendizaje (media $=1,50)$ y la Psicometría (media $=1,54)$, asignaturas todas ellas muy específicas y de elevado contenido técnico, muy propias de los estudios de Psicología en sus últimos cursos. En el extremo opuesto, la calificación media de los alumnos superó el notable en las asignaturas de Psicología Vocacional, Orientación Personal, Modelos de Comprensión Lectora y Psicología Social de la Educación, convirtiéndose, por tanto, estas cuatro materias en las «menos complicadas» del plan de estudios cursado por los sujetos de nuestra muestra, quizá por su carácter más general y su vinculación directa con ramas más puramente educativas y, por tanto, acordes con la especialización previa de nuestros estudiantes. Como puede verse, el resto de asignaturas obtuvieron calificaciones medias comprendidas en un rango que, sin alcanzar el notable, superó en todos los casos el 1,6 de media.

\section{Discusión y conclusiones}

En el apartado anterior, hemos podido constatar que, aunque los alumnos procedentes de estudios de primer grado en Lengua Extranjera y en Planes Anteriores de Magisterio parecieron obtener resultados académicos ligeramente superiores al resto de sus compañeros (especialmente a los procedentes de Educación Física y Educación Social, con las medias más bajas), no se encontraron diferencias significativas globales entre los distintos grupos.

A este respecto, tan sólo en seis de las veintiuna asignaturas comparadas en virtud de la especialización previa de nuestros estudiantes se observaron calificaciones medias estadísticamente distintas. La variedad temática de estas asignaturas y la falta de estabilidad aparente entre los grupos que en ellas divergían, no sólo complica la explicación de este hallazgo, 


\section{TABLA 2: Calificaciones medias obtenidas en las diferentes asignaturas en función de los estudios de procedencia.}

\begin{tabular}{|c|c|c|c|c|c|c|c|c|c|}
\hline & $\begin{array}{c}\text { Ed. } \\
\text { Social }\end{array}$ & $\begin{array}{c}\text { Ed. } \\
\text { Infantil }\end{array}$ & $\begin{array}{c}\text { Ed. } \\
\text { Primaria }\end{array}$ & $\begin{array}{l}\text { Aud. y } \\
\text { Lenguaje }\end{array}$ & $\begin{array}{c}\text { Ed. } \\
\text { Musical }\end{array}$ & $\begin{array}{c}\text { Planes } \\
\text { Anteriores }\end{array}$ & $\begin{array}{c}\text { Ed. } \\
\text { Especial }\end{array}$ & $\begin{array}{l}\text { Ed. } \\
\text { Física }\end{array}$ & $\begin{array}{c}\text { Lengua } \\
\text { Extranjera }\end{array}$ \\
\hline $\begin{array}{l}\text { Psicometría } \\
(\text { media }=1,54)\end{array}$ & 1,50 & 1,58 & 1,46 & 1,70 & 1,50 & 1,50 & 1,11 & 1,25 & 2,00 \\
\hline $\begin{array}{l}\text { Introducción a la Psicología } \\
(\text { media }=1,71)\end{array}$ & 1,00 & 1,79 & 1,38 & 2,15 & 1,75 & 1,75 & 1,67 & 1,33 & 1,86 \\
\hline $\begin{array}{l}\text { Psicología de la Personalidad } \\
(\text { media }=1,86)\end{array}$ & 1,50 & 1,82 & 1,88 & 2,05 & 1,88 & 2,00 & 2,00 & 1,33 & 1,86 \\
\hline $\begin{array}{l}\text { Psicología Social } \\
(\text { media }=1,93)\end{array}$ & & 2,00 & 1,81 & 2,00 & 1,88 & 2,50 & 1,89 & 1,75 & 1,86 \\
\hline $\begin{array}{l}\text { Diagnóstico en Educación } \\
(\text { media }=1,93)\end{array}$ & 1,50 & 1,91 & 2,00 & 2,05 & 2,00 & 2,00 & 2,11 & 1,33 & 2,14 \\
\hline $\begin{array}{l}\text { Dificultades de Aprendizaje } \\
(\text { media }=1,50)\end{array}$ & 1,00 & 1,38 & 1,23 & 2,10 & 1,13 & 1,67 & 1,38 & 1,80 & 1,50 \\
\hline $\begin{array}{l}\text { Modelos de Orientación } \\
(\text { media }=1,88)\end{array}$ & 1,00 & 1,82 & 1,92 & 1,90 & 2,00 & 2,67 & 2,33 & 1,00 & 2,29 \\
\hline $\begin{array}{l}\text { Métodos de Investigación } \\
(\text { media }=1,86)\end{array}$ & 2,50 & 1,71 & 2,00 & 1,81 & 1,80 & 2,33 & 2,00 & 1,33 & 1,00 \\
\hline $\begin{array}{l}\text { Psicología de la Instrucción } \\
(\text { media }=1,90)\end{array}$ & 1,50 & 2,03 & 1,96 & 1,80 & 1,86 & 2,00 & 1,67 & 1,25 & 2,57 \\
\hline $\begin{array}{l}\text { Psicología de la Intervención } \\
(\text { media }=1,97)\end{array}$ & 2,50 & 2,03 & 1,85 & 2,15 & 2,25 & 2,00 & 1,75 & 1,50 & 1,86 \\
\hline $\begin{array}{l}\text { Psicopatología de la Infancia } \\
(\text { media }=1,78)\end{array}$ & 1,00 & 1,73 & 1,58 & 1,75 & 2,00 & 2,67 & 2,11 & 1,80 & 2,00 \\
\hline $\begin{array}{l}\text { Estructura y Funcionamiento del S. E. } \\
(\text { media }=1,89)\end{array}$ & 1,50 & 1,93 & 2,09 & 1,95 & 2,50 & 1,67 & 1,89 & 0,60 & 2,50 \\
\hline $\begin{array}{l}\text { Aprendizaje Humano } \\
(\text { media }=1,23)\end{array}$ & 1,50 & 1,06 & 1,81 & 1,60 & 2,50 & 1,67 & 1,13 & 1,27 & 1,17 \\
\hline $\begin{array}{l}\text { Historia de la Educación Especial } \\
(\text { media }=1,82)\end{array}$ & & 1,83 & 1,57 & 2,00 & 1,29 & 2,00 & 3,00 & 1,00 & 1,00 \\
\hline $\begin{array}{l}\text { Psicología de la Excepcionalidad } \\
(\text { media }=1,68)\end{array}$ & & 1,50 & 2,29 & 1,53 & 1,50 & 1,00 & 2,50 & 1,50 & 1,00 \\
\hline $\begin{array}{l}\text { Psicología Clínica de la Educación } \\
(\text { media }=1,63)\end{array}$ & & 1,56 & 1,71 & 1,47 & 2,00 & 1,50 & 2,33 & 3,00 & 1,00 \\
\hline $\begin{array}{l}\text { Psicología Vocacional } \\
(\text { media }=2,03)\end{array}$ & 2,00 & 2,20 & 2,11 & 2,00 & 1,75 & 3,00 & 1,67 & 1,60 & 2,00 \\
\hline $\begin{array}{l}\text { Psicología Social de la Educación } \\
(\text { media }=2,24)\end{array}$ & 2,25 & 2,07 & 2,47 & 2,40 & 2,20 & 4,00 & 2,33 & 1,60 & 2,33 \\
\hline $\begin{array}{l}\text { Orientación Personal } \\
(\text { media }=2,07)\end{array}$ & 1,00 & 2,40 & 1,95 & 2,20 & 2,50 & 3,00 & 1,67 & 1,90 & 2,50 \\
\hline $\begin{array}{l}\text { Modelos de Comprensión Lectora } \\
(\text { media }=2,14)\end{array}$ & 2,00 & 1,94 & 2,38 & 2,20 & 2,00 & 2,33 & 2,25 & 1,58 & 2,71 \\
\hline $\begin{array}{l}\text { Etica de los Valores } \\
(\text { media }=1,82)\end{array}$ & 1,50 & 1,88 & 1,88 & 1,65 & 2,00 & 2,00 & 1,89 & 1,45 & 2,00 \\
\hline
\end{tabular}

sino que nos induce a pensar en él como en un hecho probablemente anecdótico y de escasa relevancia y consideración.

Esta inesperada homogeneidad en los datos encontrados nos hizo reflexionar sobre la propia composición de la muestra estudiada en busca de explicaciones plausibles. En pri- 
mer lugar, se nos ocurre pensar que el bajo número de alumnos existente en algunos de los subgrupos analizados (ver figura 1) podría estar restando representatividad a las calificaciones por ellos obtenidas. Además, parece más que lícito pensar que al tratarse de la primera promoción que optaba a licenciarse en el C.E.S. Don Bosco, se cuidara en extremo el proceso de preselección de los mismos, aceptándose tan sólo a aquellos alumnos con los mejores expedientes académicos de partida.

En definitiva, pensamos que ambos factores considerados de manera conjunta, podrían estar disminuyendo la «natural heterogeneidad» esperada en nuestra muestra de estudio y, con ello, resultar indetectables las diferencias que, posiblemente, pudiéramos haber encontrado en muestras más grandes, con mayor representación en los distintos subgrupos a comparar y no tan «homogeneizadas a priori» en virtud de su potencial académico.

Por todo ello, debemos concluir que:

Las calificaciones académicas de los estudiantes de primer curso Psicopedagogía del C.E.S. Don Bosco de Madrid, no presentan resultados diferenciales de consideración en función de su especialidad previa de acceso. Por tanto, no puede decirse que exista relación directa entre el resultado académico obtenido en esta licenciatura y la formación precedente por la que se accede a ellos ni que ésta última pueda utilizarse, a priori, para predecir el futuro éxito o fracaso de nuestros estudiantes de segundo ciclo.

Estudios posteriores, en los que nos planteamos incluir tanto los resultados obtenidos por las sucesivas promociones de alumnos del C.E.S., como los procedentes de distintos centros universitarios que impartan estudios de características similares al nuestro, nos permitirán contrastar definitivamente lo acertado o erróneo de las hipótesis planteadas en este trabajo y lo adecuado o inadecuado de las conclusiones que acabamos de señalar.

\section{Referencias bibliográficas}

Coll Salvador, C. (1999). Psicopedagogía: confluencia disciplinar y espacio profesional. En C. Monereo e I. Solé (coords.) El asesoramiento psicopedagógico: una perspectiva profesional y constructivista (pp.33-52). Madrid: Alianza Editorial.

Gento, S. (2000). Nuevas perspectivas de gestión educativa. XII Congreso Nacional y I Iberoamericano de Pedagogía (Sociedad Española de Pedagogía). Madrid, 26-30 de Septiembre.

Ministerio de Educación y Ciencia (1994). Centros Educativos y Calidad de Enseñanza. Madrid: MEC.

Monereo, C. y Solé, I. (1999). El modelo de asesoramiento educacional-constructivo: dimensiones críticas. En C. Monereo e I. Solé (coords.) El asesoramiento psicopedagógico: una perspectiva profesional y constructivista (pp.15-32). Madrid: Alianza Editorial.

Municio Fernández, P. (2000). Nuevos enfoques de control institucional. XII Congreso Nacional y I Iberoamericano de Pedagogía (Sociedad Española de Pedagogía). Madrid, 26-30 de Septiembre.

O.C.D.E. (1991). Escuelas y calidad de enseñanza. Informe Internacional. Madrid: Paidós / MEC.

Pérez Juste, R. (2000). La evaluación como medio para la mejora de la eficacia y de la calidad del aprendizaje, de la educación y de las instituciones. XII Congreso Nacional y I Iberoamericano de Pedagogía (Sociedad Española de Pedagogía). Madrid, 26-30 de Septiembre.

Repetto, E., Ballesteros, B. y Malik, B. (2000). Tareas y formación de los orientadores en la Unión Europea. Madrid: UNED. 


\section{Legislación educativa de referencia}

Real Decreto 916/1992, de 17 de julio, por el que se establece el título universitario oficial de Licenciado en Psicopedagogía y la aprobación de las directrices generales propias de los Planes de Estudio conducentes a la obtención de aquél (BOE 206/92 de 27 de agosto).

Orden Ministerial de 22 de diciembre de 1992, por la que se establecen las titulaciones y estudios previos del Primer Ciclo, así como los complementos de formación con los que se puede acceder a las enseñanzas de Segundo Ciclo conducentes a la obtención del título oficial de Licenciado en Psicopedagogía (BOE 11/93 de 13 de enero).

Resolución de 27 de octubre de 1994, de la Universidad Complutense de Madrid, por la que se publica el Plan de Estudios para la obtención del título oficial de Licenciado en Psicopedagogía (BOE 280/94 de 23 de noviembre).

Resolución de 23 de julio de 1998, de la Universidad Complutense de Madrid, por la que se modifica la Resolución de 27 de octubre de 1994, por la que se publica el Plan de Estudios homologado por el Consejo de Universidades para la obtención del título oficial de Licenciado en Psicopedagogía (BOE 194/98 de 14 de agosto).

Fecha de recepción: 18-III-02

Fecha de revisión: 11-VI-02

Fecha de aceptación: 5-XI-02 


\section{ANEXO}

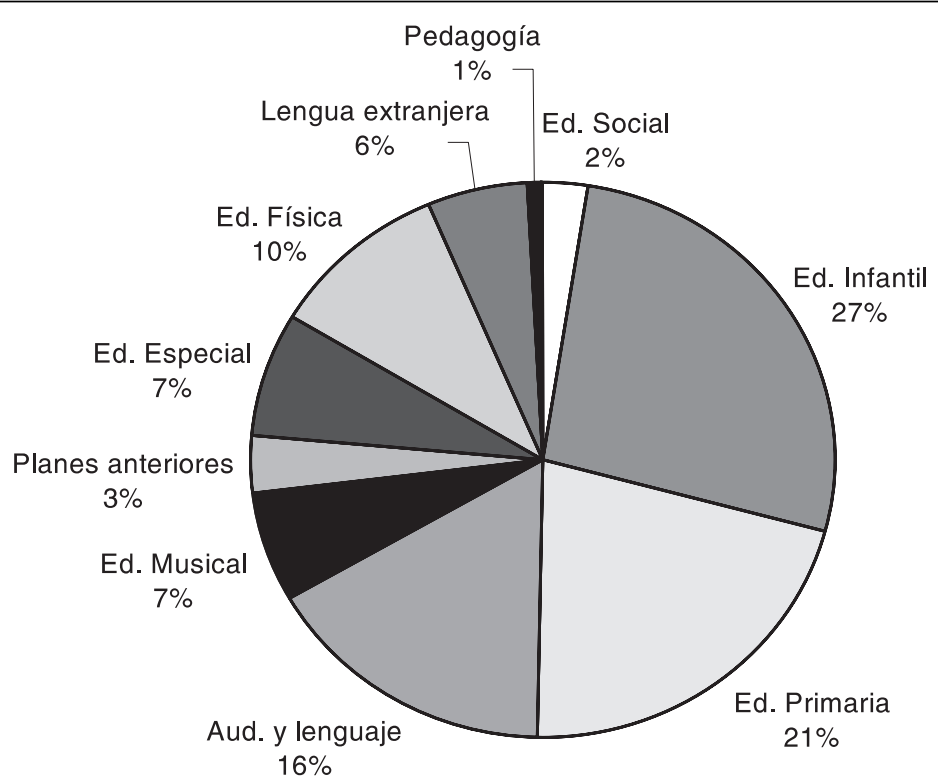

FIGURA 1.

Distribución muestral en virtud de sus estudios de procedencia.

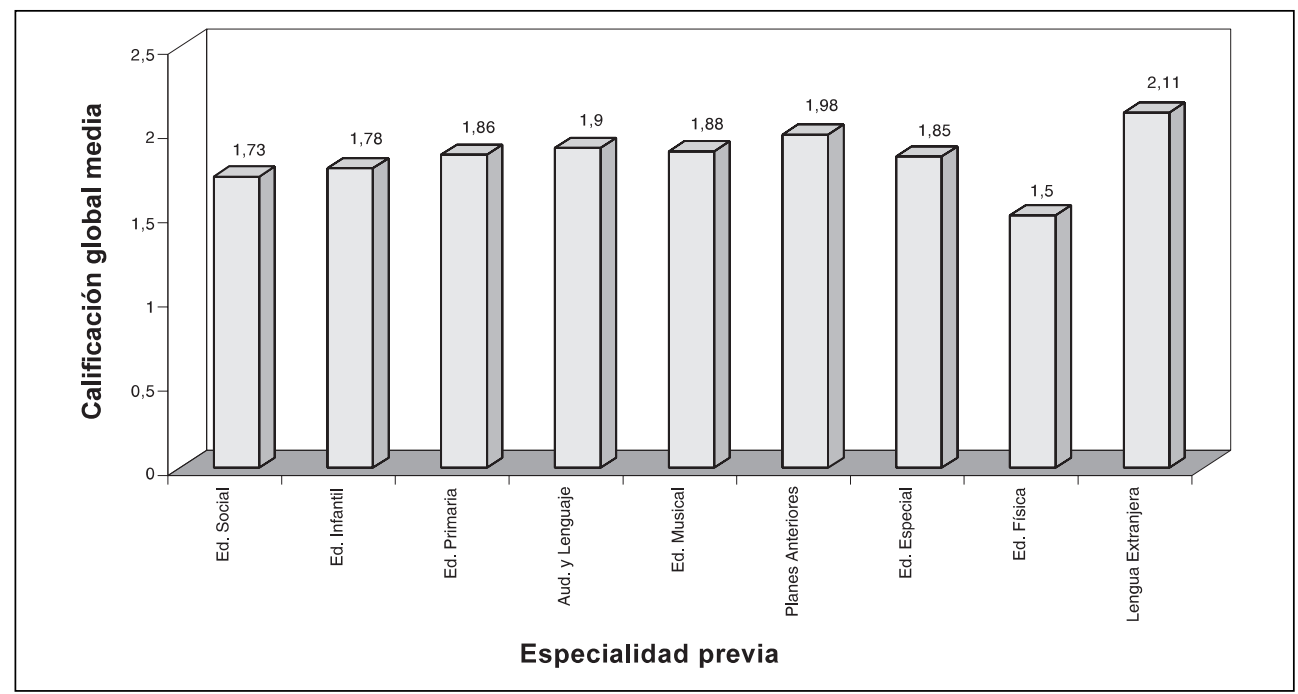

FIGURA 2.

Calificación global media en las distintas especialidades. 